F.R. Tay' ${ }^{1}$ M. Hashimoto ${ }^{2}$, D.H. Pashley ${ }^{3 *}$, M.C. Peters ${ }^{4}$, S.C.N. Lai ${ }^{\prime}$, C.K.Y. Yiu', and C.Cheong ${ }^{1}$

${ }^{1}$ Faculty of Dentistry, University of Hong Kong, Hong Kong SAR, China; ${ }^{2}$ Division of Pediatric Dentistry, Hokkaido University, Graduate School of Dental Medicine, Sapporo, Hokkaido, Japan; ${ }^{3}$ Department of Oral Biology and Maxillofacial Pathology, School of Dentistry, Medical College of Georgia, Augusta, GA 30912-1129, USA; and ${ }^{4}$ Department of Cariology, Restorative Sciences and Endodontics, School of Dentistry, University of Michigan, Ann Arbor, MI, USA; *corresponding author, dpashley@mail.mcg.edu

J Dent Res 82(7):537-541, 2003

\begin{abstract}
Water sorption into resin-dentin interfaces precedes hydrolytic degradation. We hypothesized that these processes are morphologically manifested by the uptake of ammoniacal silver nitrate, which is thought to trace hydrophilic domains and water-filled channels within matrices. Water sorption is thought to be nonuniform and can be traced by the use of silver nitrate. Human teeth bonded with an experimental filled-adhesive were aged in artificial saliva (experimental) or non-aqueous mineral oil (control). Specimens retrieved for up to a 12 month period were immersed in $50 \mathrm{wt} \%$ ammoniacal silver nitrate and examined by transmission electron microscopy for identification of the changes in their silver uptake. Reticular silver deposits initially identified within the bulk of hybrid layers in the experimental group were gradually reduced over time, but were subsequently replaced by similar deposits that were located along the hybrid layer-adhesive interface. Silver uptake in water-binding domains of the adhesive layers increased with aging, resulting in water tree formation. These waterfilled channels may act as potential sites for hydrolytic degradation of resin-dentin bonds.
\end{abstract}

KEY WORDS: water sorption, nanoleakage, water trees, hydrolytic degradation.

Received November 4, 2002; Accepted April 7, 2003

\section{Aging Affects Two Modes of Nanoleakage Expression in Bonded Dentin}

\section{INTRODUCTION}

$\mathbf{H}$ ydrolytic degradation of resin-dentin interfaces contributes to the reduction in bond strengths created by dentin adhesives over time (Watanabe and Nakabayashi, 1993; Gwinnett and Yu, 1995; Burrow et al., 1996; Shono et al., 1999; Armstrong et al., 2001). This phenomenon is aggravated by the incorporation of hydrophilic and ionic resin monomers into contemporary adhesives, since hydrophilicity and hydrolytic stability of resin monomers are generally antagonistic properties (Chiari et al., 1994; Simo-Alfonso et al., 1996). Several in vivo studies have provided morphological evidence of leaching of resin components and/or deterioration of incompletely infiltrated collagen matrices in service-aged, resin-dentin bonds (Sano et al., 1999; Hashimoto et al., 2000, 2001). However, changes in the extent of nanoleakage that occurred within hybrid layers (Sano et al., 1995; Pioch et al., 2001) did not correlate well with the time-dependent deterioration of these bonds (Okuda et al., 2001).

Hydrolytic degradation cannot occur in the absence of water uptake in bonded interfaces. Water sorption is enhanced by the presence of hydrophilic and ionic resin monomers (Burrow et al., 1999; Tanaka et al., 1999), which in turn facilitates ion movement within a polymerized resin matrix (Antonucci et al., 1996). To date, the morphological correlates of the time-dependent process of water sorption in resin-dentin interfaces have not been elucidated. Ammoniacal silver nitrate has been recently used to trace water-filled regions and/or hydrophilic polymer domains within hybrid layers and the adhesive layers in resin-bonded dentin and enamel (Tay and Pashley, 2003). These results revealed two different modes of silver tracer deposition patterns (Tay et al., 2002). We hypothesize that water sorption and, subsequently, hydrolytic degradation may be reflected by the changes in uptake of ammoniacal silver nitrate within resin-dentin interfaces. Thus, the objective of this study was to examine, with the use of transmission electron microscopy (TEM), the changes in silver uptake that occur with aging of resin-dentin bonds. The specimens were subjected to a 12-month period of aging, in the presence of unrestricted and restricted water sorption. The term "restricted" was used since it is impossible to eliminate water completely from bonded hydrated dentin without causing dehydration artifacts that are prone to artifactual silver uptake (Agee et al., 2002). The null hypothesis tested was that water sorption has no effect on the manifestation of potential water-binding domains within resin-bonded dentin.

\section{MATERIALS \& METHODS}

Ten extracted human third molars were collected after the patients' informed consent had been obtained under a protocol reviewed and approved by the institutional review board of the Medical College of Georgia, USA. Within one month of extraction, the occlusal enamel of the teeth was removed by means of a slow-speed saw (Isomet, Buehler Ltd., Lake Bluff, IL, USA) under water- 

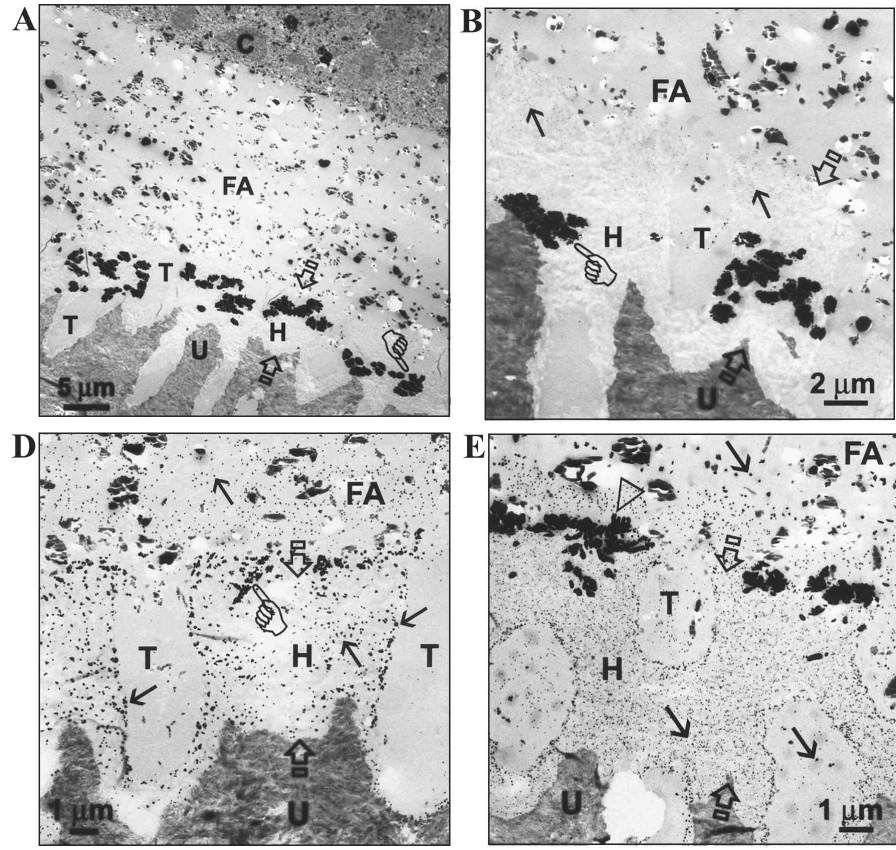

Figure 1. TEM micrographs depicting the changes in silver tracer penetration within the hybrid layers of an experimental total-etch filled adhesive after aging in artificial saliva. (A) An overall view of the resin-dentin interface in a 24-hour specimen. High-magnification views of the hybrid layers are shown after aging for: (B) 24 hrs, (C) 1 mo, (D) 3 mos, (E) 6 mos, and (F) 12 mos. Reticular silver deposits (pointers) that were initially identified within the bulk of hybrid layers were gradually reduced, and were replaced by the accumulation of similar deposits along the hybrid layer-adhesive interface. Water trees (open arrowheads) that began to form along this interface at 6 mos were clearly evident after 12 mos. There was also an increase in the size and density of the isolated silver grains (arrows) during the entire storage period. C, resin composite; FA, filled adhesive; T, dentinal tubule; U, undemineralized dentin.

cooling to create bonding surfaces in deep coronal dentin. The exposed dentin surfaces were polished with wet 180 -grit silicon carbide papers.

\section{Experimental Design}

An experimental acetone-based, water-free, two-step single-bottle filled adhesive (Lot number 387-73-A; Bisco, Inc., Schaumburg, IL, USA) that contains $15 \mathrm{wt} \%$ silanized fluoroaluminosilicate glass fillers was used for bonding to acid-etched dentin. The experimental adhesive includes three classes of resin monomers that are commonly used in dentin adhesives: a relatively hydrophobic monomer (bisphenol A diglycidyl ether dimethacrylate), a hydrophilic monomer (2-hydroxyethyl methacrylate), and an ionic monomer with carboxylic functional groups (biphenyl dimethacrylate). Camphorquinone is used as the photosensitizer, and the tertiary amine dimethylaminobenzoic acid is used as the accelerator. The rationale for using an experimental water-free adhesive was to develop a baseline of the changes in nanoleakage expression that may occur during aging of resindentin interfaces, to facilitate future evaluation of commercially available three-step total-etch, two-step total-etch and self-etch, and one-step self-etch adhesives that contain various amounts of water.

Each tooth surface was etched with a $32 \%$ phosphoric acid (Uni-Etch, Bisco, Inc.) for $15 \mathrm{sec}$ and rinsed with water for $20 \mathrm{sec}$. Two coats of the experimental adhesive were applied to the visibly moist acid-etched dentin, air-dried, and light-activated for $10 \mathrm{sec}$. A microfilled resin composite that contains pre-polymerized organic fillers (Metafil CX, Sun Medical Co. Ltd., Shiga, Japan)
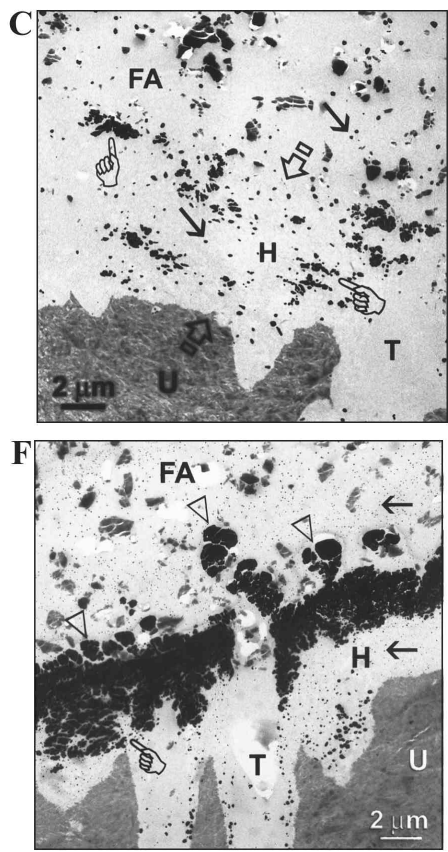

was used to facilitate ultramicrotomy. It was applied to the bonded interfaces and lightactivated in three $1-\mathrm{mm}$ increments.

After the bonds were allowed to mature for 24 hrs, each tooth was sectioned longitudinally across the resin-dentin interface into 0.9-mm-thick serial slabs. Two slabs from the center part of each tooth were used for the experiments. Ten slabs, one from each tooth, were stored in $5 \mathrm{~mL}$ of artificial saliva (Wataha et al., 1999) and incubated at $55^{\circ} \mathrm{C}$ to accelerate aging. The other 10 slabs were wiped with lint-free gauze, briefly airdried to remove excess water, and incubated at $55^{\circ} \mathrm{C}$ in $5 \mathrm{~mL}$ of oil (DowCorning 200 Fluid, DowCorning Corp., Midland, MI, USA). This served as the control group and provided a non-aqueous medium in which no additional water sorption could occur apart from the small amount that was initially present in the slabs during the period of aging and the sectioning process. The experimental specimens were examined at $24 \mathrm{hrs}, 1,3,6$, and 12 mos, while the control specimens were examined at 6 and 12 mos.

\section{Evaluation of Silver Uptake}

A $50 \mathrm{wt} \%$ ammoniacal silver nitrate solution was used as the tracer, following the silver-staining protocol of Tay et al. (2002). The rationale for using ammoniacal silver nitrate was based on the potential of the diamine silver $\left(\left[\mathrm{Ag}\left(\mathrm{NH}_{3}\right)_{2}\right]^{+}\right)$ion complex to trace water-filled regions and/or hydrophilic, water-binding polymer domains within both hybrid layers and the overlying adhesive layers (Tay and Pashley, 2003). Each slab was coated with two layers of nail varnish applied $1 \mathrm{~mm}$ from the bonded interfaces. Before the slabs could become dehydrated, they were immediately immersed in the tracer solution for $24 \mathrm{hrs}$. The silver-stained slabs were rinsed and placed in photodeveloping solution for $8 \mathrm{hrs}$ under a fluorescent light to reduce the diamine silver ions into metallic silver grains. Three blocks were prepared from each experimental slab and 2 blocks for each control slab that were retrieved at a specific time interval. Undemineralized, epoxy-resin-embedded, 90- to 100-nm-thick ultrathin sections were prepared according to the TEM protocol of Tay et al. (1999). The 35 unstained sections were examined by means of a TEM (Philips EM208S, Philips, Eindhoven, The Netherlands) operating at $80 \mathrm{kV}$.

Five images from each of the 7 groups were analyzed with image analysis software (NIH Image 1.60, Scion Corp., Frederick, MD, USA) for evaluation of the percentage distribution of silver 
deposits within the hybrid layers. Due to the interference from glass fillers, the distribution of silver deposits in the adhesive layers was not determined. Data were statistically analyzed by Kruskal-Wallis ANOVA on ranks and Dunn's multiple comparison tests with statistical significance set at $\alpha=0.05$.

\section{RESULTS}

Two modes of nanoleakage expression could be recognized within resin-dentin interfaces: reticular and spotted types. Both of these patterns exhibited substantial changes when specimens were aged in artificial saliva, but to a much slower extent when specimens were aged in
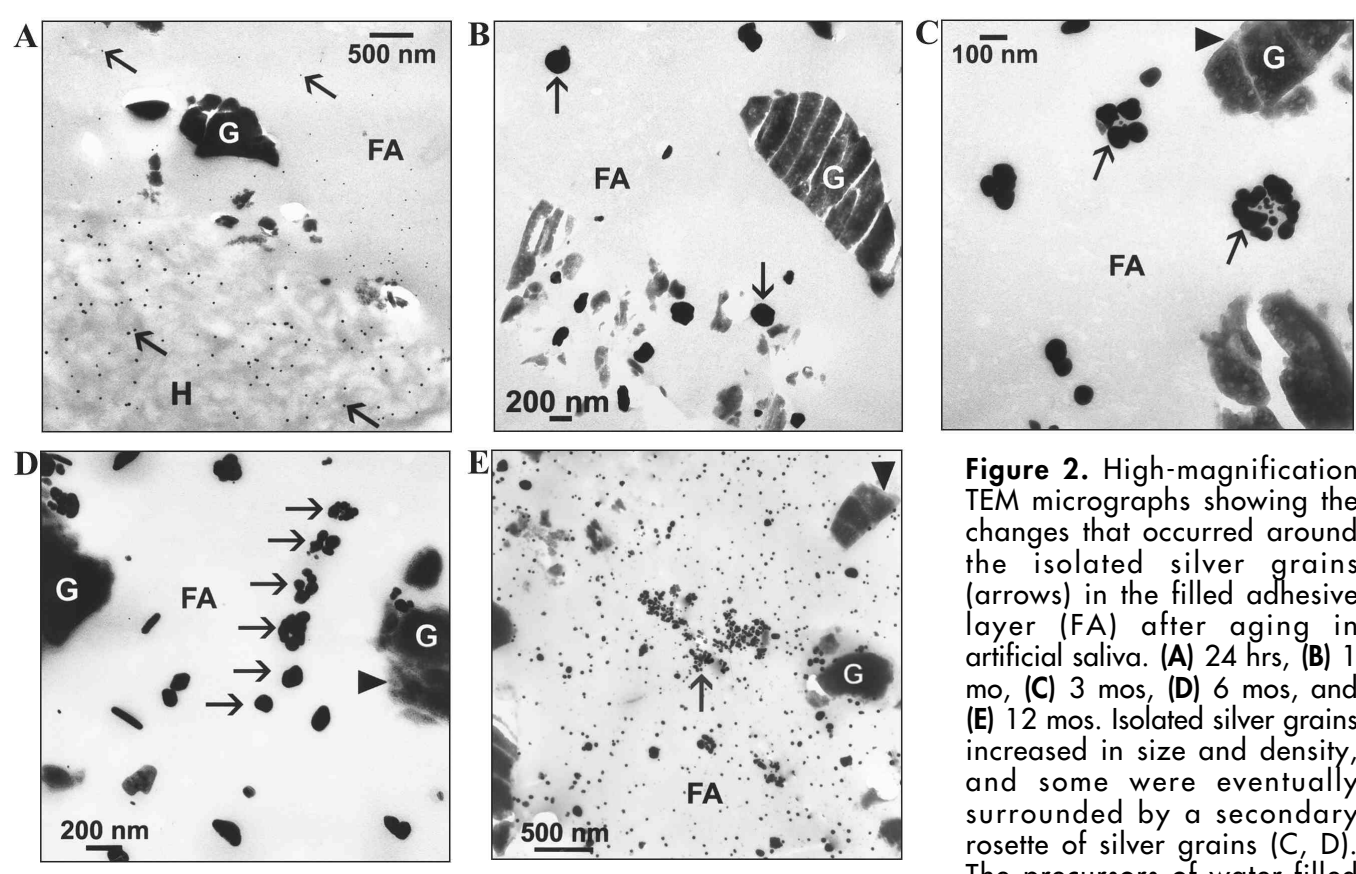

Figure 2. High-magnification TEM micrographs showing the changes that occurred around the isolated silver grains (arrows) in the filled adhesive layer (FA) after aging in artificial saliva. (A) $24 \mathrm{hrs,} \mathrm{(B)} 1$ mo, (C) 3 mos, (D) 6 mos, and (E) 12 mos. Isolated silver grains increased in size and density, and some were eventually surrounded by a secondary rosette of silver grains $(C, D)$. The precursors of water-filled channels (i.e., water trees) that could be seen at 6 mos were attributed to the increase in density of these rosettes of silver grains. Frank water trees were evident at 12 mos (E). A siliceous hydrogel layer (arrowheads) could also be observed along the periphery of the fluoroaluminosilicate glass fillers $(G)$ beginning at 3 mos.

oil. In specimens that

were aged in artificial

saliva, the reticular type consisted originally of discontinuous islands of silver deposits that were randomly distributed within the bulk of the unstained hybrid layers (Fig. 1A). These incompletely resin-infiltrated zones (Fig. 1B) gradually reduced in their dimensions at 1 mo (Fig. 1C) until they became truly reticular in appearance by the end of 3 mos (Fig. 1D). While the reticular silver deposits within the hybrid layer continued to diminish with time, there was a gradual accumulation of similar silver deposits along the hybrid layer-adhesive interface (Fig. $1 \mathrm{E})$, until they occupied almost the entire top half of the hybrid layer at 12 mos (open arrowheads, Fig. 1F). Water trees (i.e., water-filled channels; Tay and Pashley, 2003) that began to form along this interface at 6 mo (Fig. 1E) became clearly evident at 12 mos (Fig. 1F). There was also an increase in the size and density of the spotted type of nanoleakage (i.e., isolated silver grains) within the hybrid layers during the entire storage period.

High-magnification views of these specimens also revealed the existence of isolated silver grains within the adhesive layers of the resin-dentin interfaces. At $24 \mathrm{hrs}$, only very fine grains could be identified within the filled adhesive and hybrid layers (Fig. 2A). They increased in size and density at 1 mo (Fig. 2B). At 3 mos, additional rosettes of silver grains were formed around some of the original silver grains (Fig. 2C). Precursors of water trees that were created by the aggregation of the silver rosettes could be seen at 6 mos (Fig. 2D). Frank water trees were evident in the adhesive layers at 12 mos (Fig. 2E).

For specimens that were aged in oil for 6 mos (Fig. 3A), the original discontinuous islands of silver deposits within the hybrid layers were similar in dimension to those in the 24-hour artificial saliva specimens (Fig. 1B). However, a more extensive distribution of isolated silver grains could be identified. The bulk silver deposits within the hybrid layers were considerably diminished at 12 mos and appeared as truly reticular patterns (Fig. 3B). The dimensions of these reticular patterns (Fig. 3C) were comparable with those of specimens that were stored in artificial saliva after 1 mo (Fig. 1C). Fine, sparsely distributed isolated silver grains could also be observed in the adhesive layers of the 12-month mineral oil specimens (Fig. 3D). Features such as rosette formation and water trees that were identified from the experimental specimens were absent from the control specimens stored in oil.

Since we could not differentiate between the two modes of nanoleakage expression during image analyses, only the total percentage distributions of silver tracer within the hybrid layers are shown in Fig. 4. The 12-month artificial saliva group has a significantly higher silver tracer distribution than do the onemonth artificial saliva group and the 12 -month oil group $(\mathrm{P}<$ $0.05)$.

\section{DISCUSSION}

Since the two modes of nanoleakage expression were affected by aging of resin-dentin bonds, this led to a rejection of the null hypothesis. The changes observed provided a working model for future comparisons of commercially available three-step, two-step, and one-step dentin adhesives. Some of these changes were observed in both the experimental and control specimens, such as the initial reduction of the reticular silver tracer patterns within the bulk of the hybrid layer, and the increase in size and density of the isolated silver grains within the adhesive. However, these features were manifested much more slowly when the control specimens were aged in a non-aqueous medium. This may be attributed to restricted water uptake from the minimal amount of water that was initially trapped within 

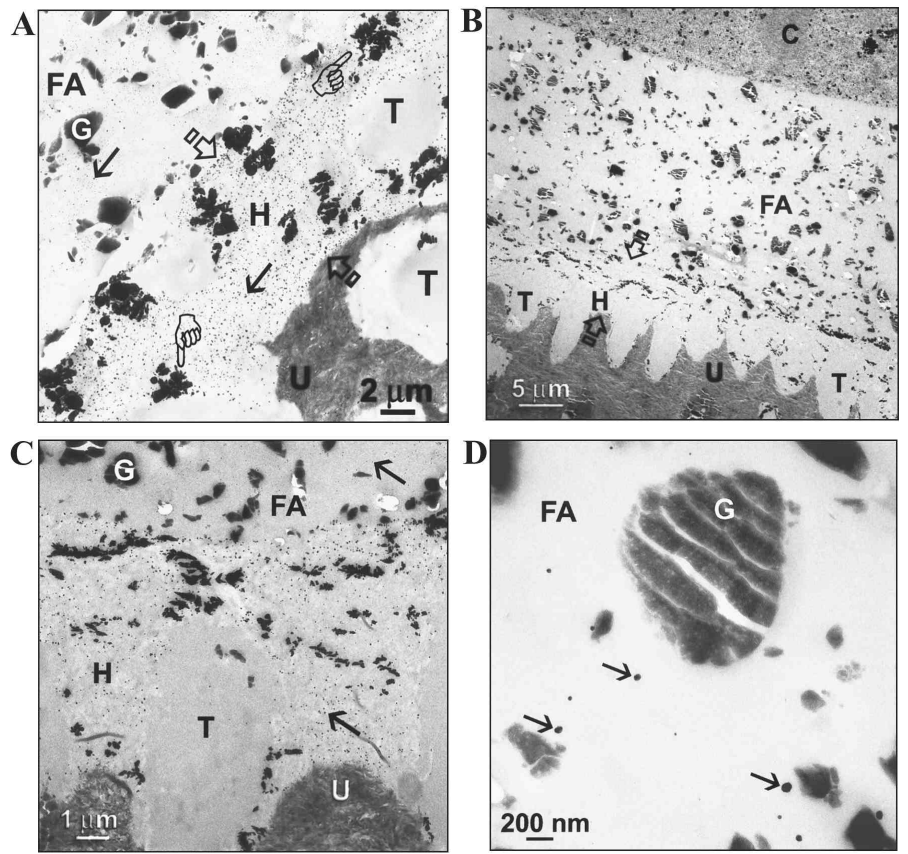

Figure 3. TEM micrographs showing the changes in silver tracer penetration along the resin-dentin interfaces after aging in oil (control). (A) 6 mos, (B-D) 12 mos. Pointer, reticular patterns of silver deposits; arrow, isolated silver grains; $\mathrm{C}$, resin composite; $\mathrm{FA}$, filled adhesive; $\mathrm{H}$, hybrid layer; $\mathrm{T}$, dentinal tubule; $U$, undemineralized dentin; $G$, fluoroaluminosilicate glass fillers. (A) Up to 6 mos, islands of heavy silver deposits could still be seen in the hybrid layers. (B) An overall view of a specimen examined after aging in oil for 12 mos revealed a considerable reduction of these bulk deposits to reticular silver patterns. (C) A high-magnification view of the hybrid layer in the 12month specimen, showing that the extent of the reticular silver deposits within the hybrid layer was similar to that in the one-month specimen that was stored in artificial saliva (Fig. 1C). (D) A high-magnification view of the adhesive layer in the 12-month specimen revealed fine, sparsely distributed isolated silver grains that were devoid of rosettes or water tree formation.

the specimens by the oil.

The transition from the original isolated silver grains to frank water trees in the adhesive resin matrices may be viewed as a series of events that is initiated by water sorption. Water that is retained within hydrophilic polymers via hydrogen bonding exits as a spectrum of intermediate states, ranging from strongly bound primary hydration spheres, to less-ordered hydrated water associated in the form of secondary hydration shells, to free water that appears as water-filled channels (Hamilton et al., 1988; Zaikov et al., 1988). Depending on the state of water that exists, water movement in polymer membranes may begin as a slow Fickian diffusion-type mechanism through the entire matrix, and then become more rapid as convective transport routes develop through the isolated water-filled channels (Zaikov et al., 1988; Steward et al., 1995). Thus, the original isolated silver grains may represent sites where water molecules are structurally bound as hydration spheres to the polar and ionic functional groups of the hydrophilic adhesive. The rosettes of silver grains that were subsequently formed around the original silver grains (Fig. 2C) may represent the secondary hydration shells that, with time, may coalesce and form the water-filled channels (water trees) within the adhesive. In a previous study, water trees were observed only in water-containing adhesives when resin-dentin interfaces were examined immediately after bonding (Tay and

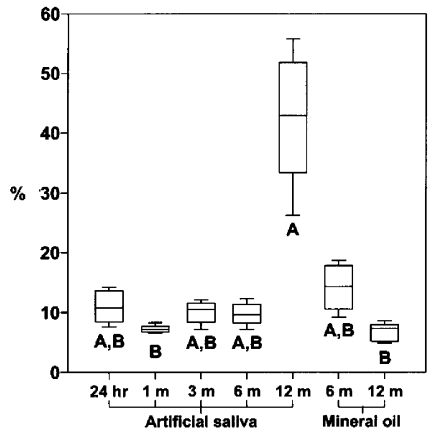

Figure 4. Percentage distribution of silver deposits within the hybrid layers of specimens aged in different media for up to 12 mos. Both modes of silver deposition in the hybrid layers are included, since we could not distinguish between the reticular patterns and the isolated silver grains during image analyses. Groups identified by different letter are significantly different $(p<$

0.05). $M$, month. In box plots, the distance between the upper and lower brackets includes $80 \%$ of the values. The box includes $50 \%$ of the values. The horizontal line in the box represents the median.

Pashley, 2003). This study further demonstrated that water trees may emerge with time in an aged water-free adhesive that is initially devoid of these water-conducting channels. This water must be slowly absorbed and accounts for the increased porosities observed in adhesive layers after in vivo aging (Sano et al., 1999; Takahashi et al., 2002). Unlike the original silver grains that were segregated from one another, unrestricted water movement within the water trees along the hybrid layer-adhesive interface probably permits a more rapid rate of resin leaching to occur and expedites hydrolytic degradation along these channels.

The significant increase in silver tracer that extended from the hybrid layer-adhesive interface into the hybrid layer in the 12-month experimental specimens supported the findings of Li et al. (2001) and may be interpreted as the morphological manifestation of hydrolytic degradation of resin-infiltrated hybrid layers. Unlike the reticular silver patterns seen within the hybrid layers of the 24-hour experimental specimens that were the original sites of incomplete resin infiltration, the highly concentrated reticular pattern of silver did not appear until the isolated silver grains had increased in size and density, sometime between 6 and 12 mos. This is a reasonable expectation, since water sorption should precede hydrolytic degradation. Expansion of the resin within the hybrid layer, caused by water sorption, seems to have started in the lower half of the hybrid layer and slowly closed those porosities but opened up others closer to the top of the hybrid layer after 1 mo of aging in artificial saliva and at the top by 6 mos. A similar trend was also observed in specimens that were aged in oil, albeit to a much smaller extent. Such a phenomenon supports the absence of correlation between incompletely infiltrated hybrid layers and the decline in resin-dentin bond strengths over time (Okuda et al., 2001) seen in total-etch adhesives. Since the original sites of incomplete resin infiltration may not correspond to the sites of hydrolytic degradation, this finding complements those of Hashimoto et al. (2002), who reported that resins were gradually extracted from the hybrid layer-adhesive interface when exposed dentin specimens were aged in water for 12 mos. It is possible that, as resins continue to leach components via the water trees along this interface, endogenous matrix metalloproteinases such as MMP-2, -8, -9, and -20 (BirkedalHansen et al., 1993) are slowly released from the denuded 
demineralized dentin matrix, further contributing to the degradation of the hybrid layer in a way that is similar to caries progression (Tjäderhane et al., 1998; Sulkala et al., 2002). This hypothesis merits further investigation by aging exposed dentin in an aqueous medium that contains protease inhibitors.

\section{ACKNOWLEDGMENTS}

We thank Bisco, Inc. for producing the experimental batch of filled One-Step adhesive used in this study. The resin composite was generously sponsored by Sun Medical Co. Ltd. This study was supported by grant DE 014911 from the National Institute of Dental and Craniofacial Research (Bethesda, MD, USA). The authors are grateful to Michelle Barnes for secretarial support and Amy Wong for technical support.

\section{REFERENCES}

Agee KL, Pashley EL, Itthagarun A, Sano H, Tay FR, Pashley DH (2002). Submicron hiati in acid-etched dentin are artifacts of desiccation. Dent Mater 19:60-68.

Antonucci JM, Eanes ED, Skrtic D (1996). Polymeric amorphous calcium phosphate compositions. United States Patent No. $5,508,342$.

Armstrong SR, Keller JC, Boyer DB (2001). The influence of water storage and $\mathrm{C}$-factor on the dentin-resin composite microtensile bond strength and debond pathway utilizing a filled and unfilled adhesive resin. Dent Mater 17:268-276.

Birkedal-Hansen H, Moore WG, Bodden MK, Windsor LJ, BirkedalHansen B, DeCarlo A, et al. (1993). Matrix metalloproteinases: a review. Crit Rev Oral Biol Med 4:197-250.

Burrow MF, Satoh M, Tagami J (1996). Dentin bond durability after three years using a dentin bonding agent with and without priming. Dent Mater 12:302-307.

Burrow MF, Inokoshi S, Tagami J (1999). Water sorption of several bonding resins. Am J Dent 12:295-298.

Chiari M, Micheletti C, Nesi M, Fazio M, Righetti PG (1994). Towards new formulations for polyacrylamide matrices: Nacryloylaminoethoxyethanol, a novel monomer combining high hydrophilicity with extreme hydrolytic stability. Electrophoresis 15:177-186.

Gwinnett AJ, Yu S (1995). Effect of long-term water storage on dentin bonding. Am J Dent 8:109-111.

Hamilton CJ, Murphy SM, Atherton ND, Tighe BJ (1988). Synthetic hydrogels: 4. The permeability of poly(2-hydroxyethyl methacrylate) to cations - an overview of solute-water interactions and transport processes. Polymer 29:1879-1886.

Hashimoto M, Ohno H, Kaga M, Endo K, Sano H, Oguchi H (2000). in vivo degradation of resin-dentin bonds in humans over 1 to 3 years. J Dent Res 79:1385-1391.

Hashimoto M, Ohno H, Kaga M, Endo K, Sano H, Oguchi H (2001). Resin-tooth adhesive interfaces after long-term function. Am J Dent 14:211-215.

Hashimoto M, Ohno H, Sano H, Tay FR, Kaga M, Kudou Y, et al. (2002). Micromorphological changes in resin-dentin bonds after 1 year of water storage. J Biomed Mater Res 63:306-311.
Li HP, Burrow MF, Tyas MJ (2001). The effect of long-term storage on nanoleakage. Oper Dent 26:609-616.

Okuda M, Pereira PN, Nakajima M, Tagami J (2001). Relationship between nanoleakage and long-term durability of dentin bonds. Oper Dent 26:482-490.

Pioch T, Staehle HJ, Duschner H, García-Godoy F (2001). Nanoleakage at the composite-dentin interface: a review. Am J Dent 14:252-258.

Sano H, Yoshiyama M, Ebisu S, Burrow MF, Takatsu T, Ciucchi B, et al. (1995). Comparative SEM and TEM observations of nanoleakage within the hybrid layer. Oper Dent 20:160-167.

Sano H, Yoshikawa T, Pereira PN, Kanemura N, Morigami M, Tagami $\mathrm{J}$, et al. (1999). Long-term durability of dentin bonds made with a self-etching primer, in vivo. J Dent Res 78:906-911.

Shono Y, Terashita M, Shimada J, Kozono Y, Carvalho RM, Russell CM, et al. (1999). Durability of resin-dentin bonds. J Adhes Dent 1:211-218.

Simo-Alfonso E, Gelfi C, Sebastiano R, Citterio A, Righetti PG (1996). Novel acrylamido monomers with higher hydrophilicity and improved hydrolytic stability: II. Properties of Nacryloylaminopropanol. Electrophoresis 17:732-737.

Steward PA, Hearn J, Wilkinson MC (1995). Studies in permeation through polymer latex films. III. Modification using soluble polymeric additives. Polymer Int 38:23-32.

Sulkala M, Larmas M, Sorsa T, Salo T, Tjäderhane L (2002). The localization of matrix metalloproteinase-20 (MMP-20, enamelysin) in mature human teeth. J Dent Res 81:603-607.

Takahashi A, Inoue S, Kawamoto C, Ominato R, Tanaka T, Sato Y, et al. (2002). in vivo long-term durability of the bond to dentin using two adhesive systems. $J$ Adhes Dent 4:151-159.

Tanaka J, Ishikawa K, Yatani H, Yamashita A, Suzuki K (1999). Correlation of dentin bond durability with water absorption of bonding layer. Dent Mater J 18:11-18.

Tay FR, Pashley DH (2003). Water treeing-a potential mechanism for degradation of dentin adhesives. Am J Dent 16:6-12.

Tay FR, Moulding KM, Pashley DH (1999). Distribution of nanofillers from a simplified-step adhesive in acid conditioned dentin. $J$ Adhes Dent 1:103-117.

Tay FR, Pashley DH, Yoshiyama M (2002). Two modes of nanoleakage expression in single-step adhesives. $J$ Dent Res 81:472-476.

Tjäderhane L, Larjava H, Sorsa T, Uitto VJ, Larmas M, Salo T (1998). The activation and function of host matrix metalloproteinases in dentin matrix breakdown in caries lesions. J Dent Res 77:16221629 .

Wataha JC, Rueggeberg FA, Lapp CA, Lewis JB, Lockwood PE, Ergle JW, et al. (1999). In vitro cytotoxicity of resin-containing restorative materials after aging in artificial saliva. Clin Oral Investig 3:144-149.

Watanabe I, Nakabayashi N (1993). Bonding durability of photocured phenyl-P in TEGDMA to smear layer-retained bovine dentin. Quintessence Int 24:335-342.

Zaikov GE, Iordanskii AL, Markin VS (1988). Diffusion of electrolytes in polymers. Utrecht, The Netherlands: VSP BV (formerly VNU Science Press BV), pp. 48-70. 